Bull. Korean Math. Soc. 48 (2011), No. 3, pp. 455-468

DOI 10.4134/BKMS.2011.48.3.455

\title{
REFINEMENT RINGS, EXCHANGE PROPERTY AND COMPARABILITY
}

\author{
Chaoling Huang
}

\begin{abstract}
We introduce the notion of refinement rings, and generalize some comparability properties of regular rings to the setting of refinement rings.
\end{abstract}

\section{Introduction}

Recall that a monoid $(\mathbb{M},+, 0)$ is a nonempty set $\mathbb{M}$ with a binary operation + which is associative and which has an identity 0 such that $a=a+0=0+a$ for all $a \in \mathbb{M}$. Let $R$ be an associative ring with identity. Let $F P(R)$ denote the category of finitely generated projective right $R$-modules. Let $V(R)=\{[A] \mid[A]$ is the class of $R$-modules isomorphic to $A, A \in F P(R)\}$. Define $[A]+[B]=$ $[A \oplus B]$ for $[A],[B] \in V(R)$. Then $V(R)$ is a commutative monoid. Recall that a monoid $\mathbb{M}$ has refinement if for all $a_{1}, a_{2}, b_{1}, b_{2} \in M$ with $a_{1}+a_{2}=b_{1}+b_{2}$, there exist $c_{11}, c_{12}, c_{21}, c_{22} \in M$ such that

$$
\begin{array}{ll}
a_{1}=c_{11}+c_{12}, & a_{2}=c_{21}+c_{22}, \\
b_{1}=c_{11}+c_{21}, & b_{2}=c_{12}+c_{22} .
\end{array}
$$

Note that for a positive integer $s$, a monoid $\mathbb{M}$ is said to satisfy $s$-comparability if for any $a, b \in \mathbb{M}$, either $a$ is a summand of $s b$, or $b$ is a summand of $s a$. In [8, p. 275], a von Neumann regular ring $R$ is said to satisfy $s$-comparability in case for any $x, y \in R$, either $x R$ is isomorphic to a summand of $s(y R)$, or $y R$ is isomorphic to a summand of $s(x R)$. For the regular ring $R, R$ satisfies $s$-comparability if and only if $V(R)$ satisfies $s$-comparability. A monoid $\mathbb{M}$ is said to be separative if for any $a, b \in \mathbb{M}, a+a=a+b=b+b$ implies that $a=b$. On the other hand, $R$ is said to be separative if the following condition holds for all $A, B \in F P(R)$ :

$$
A \oplus A \cong A \oplus B \cong B \oplus B \Rightarrow A \cong B .
$$

Received March 16, 2009; Revised December 2, 2010.

2010 Mathematics Subject Classification. 16D70, 16E50.

Key words and phrases. refinement ring, exchange ring, exchange property, almost comparability. 
It is obvious that $R$ is separative if and only if $V(R)$ is separative. It was proved that a conical $(x+y=0$ implies that $x=y=0$ for any $x, y \in \mathbb{M})$ refinement monoid $\mathbb{M}$ satisfying $s$-comparability is separative [14, Theorem 1.9]. For the ring theory, we know that any exchange ring (see the following definition) satisfying $s$-comparability is separative [14, Theorem 2.2].

For any right $R$-module $M$, Crawley and Jónsson [7] called $M$ to have the exchange property if for every right $R$-module $A$ and any two decompositions of $A$,

$$
A=M^{\prime} \oplus N=\oplus_{i \in I} A_{i},
$$

where $M^{\prime} \cong M$, there are submodules $A_{i}^{\prime} \subseteq A_{i}$ such that $A=M^{\prime} \oplus\left(\oplus_{i \in I} A_{i}^{\prime}\right)$. It follows from the modular law that $A_{i}^{\prime}$ must be a direct summand of $A_{i}$ for all $i$. Warfield [15] called a ring $R$ an exchange ring if $R$ has the exchange property as a right $R$-module. He proved that this definition is left-right symmetric [15, Corollary 2]. Many classes of rings belong to this class of rings, for instance, local rings, von Neumann regular rings, semiperfect rings and strongly $\pi$-regular rings, etc.

The notion of almost comparability for regular rings was first introduced by Ara and Goodearl [2], for giving an alternative proof of the outstanding O'Meara's Theorem: directly finite simple regular rings with weak comparability are unit-regular. It was proved that for a regular $\operatorname{ring} R, R$ satisfies almost comparability if and only if every finitely generated projective $R$-module satisfies almost comparability [12, Theorem 1.9], and that almost comparability is Morita invariant [12, Theorem 1.11]. O'Meara first introduced the notion of weak comparability, and proved that simple directly finite regular rings with weak comparability must be unit-regular [8, Open Problem 3]. Many authors studied regular rings with weak comparability $[6,10,11]$. For the regular ring $R$ with weak comparability, it was proved [11, Theorem 1.6] that $A \oplus C \prec B \oplus C$ implies $A \prec B$ for any finitely generated projective $R$-modules $A, B$ and $C$ with $B \neq 0$, and was proved [11, Theorem 1.8] that $n A \prec n B$ implies $A \prec B$ for any positive integer $n$ and any finitely generated projective $R$-modules $A$ and $B$. In this note we define the notion of refinement rings, and give some generalizations of comparability from regular rings to refinement rings, especially exchange rings.

Throughout this note, $R$ is an associative ring with identity and $R$-modules are unitary right $R$-modules.

\section{Refinement rings with almost comparability}

Notation 1.1. For two $R$-modules $M$ and $N$, we use $M \lesssim \oplus N$ (respectively $M \lesssim N$ ) to denote that $M$ is isomorphic to a direct summand of $N$ (respectively $M$ is isomorphic to a submodule of $N$ ), and $M \prec_{\oplus} N$ (respectively $M \prec N$ ) to denote that $M$ is isomorphic to a proper direct summand of $N$ (respectively $M$ is isomorphic to a proper submodule of $N$ ). Let $M$ and $N$ be finitely generated projective $R$-modules. We write $M \lesssim_{a} N$ to mean that for any 
nonzero principal right ideal $C$ of $R, M \lesssim_{\oplus} N \oplus C$, and $M \prec_{a} N$ to mean that for any nonzero principal right ideal $C$ of $R, M \prec_{\oplus} N \oplus C$. Other basic notations can be found in [8].

It is well-known that the concepts of almost comparability and $s$-comparability were defined over regular rings originally. Since we consider more generally the case, we give the following definition.

Definition 1.2. A ring $R$ is said to satisfy almost comparability, if for $x, y \in R$

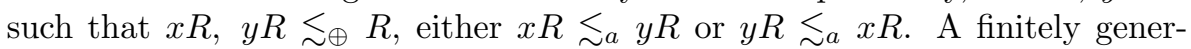
ated projective $R$-module $P$ satisfies almost comparability, if for any direct summands $A, B$ of $P$ either $A \lesssim_{a} B$ or $B \lesssim_{a} A$. Similarly, we can define the strictly almost comparability of $R$ if $x R \prec_{a} y R$ or $y R \prec_{a} x R$, and strictly almost comparability of $P$ if $A \prec_{a} B$ or $B \prec_{a} A$. Clearly, almost comparability for modules is inherited by direct summands, and $R$ satisfies almost comparability if and only if so does the $R$-module $R_{R}$. For a positive integer $s, R$ is said to satisfy $s$-comparability if, for any right ideals $A, B$ such that $A, B \lesssim_{\oplus} R$, either $A \lesssim_{\oplus} s B$ or $B \lesssim_{\oplus} s A$. It is clear that almost comparability implies 2comparability. We say that the finitely generated projective $R$-modules satisfy $s$-comparability if for any two finitely generated projective $R$-modules $A$ and $B$, either $A \lesssim_{\oplus} s B$ or $B \lesssim_{\oplus} s A$.

Definition 1.3. Let $R$ be a ring. If $V(R)$ has refinement, i.e., for finitely generated projective right $R$-modules $A_{1}, A_{2}, B_{1}, B_{2}, A_{1} \oplus A_{2} \cong B_{1} \oplus B_{2}$ implies that there exist $R$-modules $C_{i j}, i, j=1,2$ such that

$$
A_{i}=C_{i 1} \oplus C_{i 2}, \quad B_{j} \cong C_{1 j} \oplus C_{2 j}, i, j=1,2,
$$

we call $R$ a refinement ring.

It is convenient to record refinement with the following matrix:

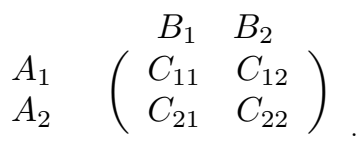

By [4, Proposition 1.1], the class of exchange rings is included in the class of the refinement rings. There are lots of refinement rings, not necessarily exchange rings, for instance $R=K\left[x_{1}, \ldots, x_{n}\right]$, where $K$ is a field. In fact, by famous Quillen-Suslin Theorem, every finitely generated projective $R$-module is free. Thus $R$ is a refinement ring. Since the idempotent elements of $R$ are those of $K, R$ is not exchange. We also argue that there exists a refinement ring with almost comparability which is not von Neumann regular. Let $A_{n}=\mathbb{M}(\mathbb{C})_{2^{n}}$ be the $C^{*}$-algebra of $2^{n} \times 2^{n}$ complex matrices. Define $h_{n}: A_{n} \rightarrow A_{n+1}$ by $h_{n}(a)=\operatorname{diag}(a, a)$. Let $A=\lim _{n \rightarrow \infty}\left(A_{n}, h_{n}\right)$ be the completion of $A^{\prime} / p^{-1}(0)$ which is called the inductive limit of the sequence $\left(A_{n}, h_{n}\right)$, where $A^{\prime}=\left\{\left\{a_{n}\right\} \in\right.$ $\prod_{n=1}^{\infty} A_{n}: a_{n+1}=h_{n}\left(a_{n}\right)$ for all sufficiently large $\left.n\right\}$ and $p(a)=\lim _{n \rightarrow \infty}\left\|a_{n}\right\|$ (see [13, p. 53]). $A$ is an infinite dimensional simple unital $A F$-algebra (see 
[13, Example 1.10.8]). Thus, by [13, Corollary 3.2.2], $A$ has real rank zero. Hence $A$ is a refinement ring by [6, Lemma 2.3]. Moreover, by [13, Chapter 3 , 3.3 and 3.4], we know that $A$ satisfies almost comparability. But $A$ is not von Neumann regular by [9, Theorem, p. 58].

Lemma 1.4. Let $R$ be a refinement ring, and $A_{i}, B_{j}$ be finitely generated projective right $R$-modules for $1 \leq i \leq n, 1 \leq j \leq m$, if $A_{1} \oplus A_{2} \oplus \cdots \oplus A_{n} \lesssim \oplus$ $B_{1} \oplus B_{2} \oplus \cdots \oplus B_{m}$, then

(1) there exist decompositions $A_{i}=A_{i 1} \oplus \cdots \oplus A_{i m}$ for $i=1, \ldots, n$ such that $A_{1 j} \oplus \cdots \oplus A_{n j} \lesssim_{\oplus} B_{j}$ for $j=1, \ldots, m$.

(2) there exist decompositions $B_{j}=B_{j 1} \oplus \cdots \oplus B_{j n}$ for $j=1, \ldots, m$ such that $A_{i} \cong B_{1 i} \oplus \cdots \oplus B_{m i}$ for $i=1, \ldots, n-1$, and $A_{n} \lesssim_{\oplus} B_{1 n} \oplus \cdots \oplus B_{m n}$.

The lemma below was obtained by Ara and Goodearl over regular rings, see [2, Lemma 3.3].

Lemma 1.5. Let $A, B$ be finitely generated projective right modules over a refinement ring $R$. If $A \lesssim_{\oplus} n$ for some positive integer $n$, then there is a decomposition $A=A_{1} \oplus A_{2} \oplus \cdots \oplus A_{n}$ such that $A_{1} \lesssim_{\oplus} A_{2} \lesssim_{\oplus} \cdots \lesssim_{\oplus} A_{n} \lesssim \oplus B$.

Proof. We prove it by the induction. By Lemma 1.4, there is a decomposition $A=U \oplus W$, where $U \lesssim_{\oplus}(n-1) B$, and $W \lesssim_{\oplus} B$. By the induction, there is a chain $C_{1} \subseteq C_{2} \subseteq \cdots \subseteq C_{n-1}$ of $B$ such that $U \cong C_{1} \oplus C_{2} \oplus \cdots \oplus C_{n-1}$. Let $D_{1}, \ldots, D_{n}$ be submodules of $B$ such that $D_{1}=C_{1}, C_{i}=C_{i-1} \oplus D_{i}$ for $i=2, \ldots, n-1$ and $B=C_{n-1} \oplus D_{n}$. Then $B=D_{1} \oplus D_{2} \oplus \cdots \oplus D_{n}$. By Lemma 1.4, it follows from $W \lesssim_{\oplus} B$ that there are submodules $X_{i} \subseteq D_{i}, i=1, \ldots, n$ such that $W \cong X_{1} \oplus X_{2} \oplus \cdots \oplus X_{n}$. Hence $A=U \oplus W \cong C_{1} \oplus C_{2} \oplus \cdots \oplus$ $C_{n-1} \oplus X_{1} \oplus X_{2} \oplus \cdots \oplus X_{n}=X_{1} \oplus\left(C_{1} \oplus X_{2}\right) \oplus \cdots \oplus\left(C_{n-1} \oplus X_{n}\right)$, where $X_{1} \subseteq\left(C_{1} \oplus X_{2}\right) \subseteq \cdots \subseteq\left(C_{n-1} \oplus X_{n}\right) \subseteq B$. Set $A_{1}=X_{1}, A_{i}=\left(C_{i-1} \oplus X_{i}\right)$ for $i=2, \ldots, n$, as desired.

Now we argue that $s$-comparability of refinement rings is inherited by finitely generated projective right $R$-modules, which is similar to a result about regular rings [5, Proposition 2.1]. So the idea of the following proposition is due to Ara, O'Meara and Tyukavkin.

Proposition 1.6. Let $R$ be a refinement ring satisfying s-comparability. Then the finitely generated projective right $R$-modules also satisfy s-comparability.

Proof. Let $A, B$ be finitely generated projective right $R$-modules. There is a positive number $n$ such that $A, B \lesssim \oplus n R$. We can prove the assertion by induction on $n$. If $n=1$, it is true since $R$ satisfies $s$-comparability. Assume that the assertion holds for $n-1$ and suppose $A, B \lesssim \oplus n R$. Since $R$ is a refinement ring, by Lemma 1.4, we can write that $A=A_{1} \oplus A_{2}$ and $B=B_{1} \oplus B_{2}$ where $A_{i}, B_{i} \lesssim \oplus(n-1) R$ for $i=1,2$. By the induction, we have $A_{1} \lesssim \oplus s B_{1}$ or $B_{1} \lesssim \oplus s A_{1}$, and $A_{2} \lesssim \oplus s B_{2}$ or $B_{2} \lesssim_{\oplus} s A_{2}$. If $A_{1} \lesssim_{\oplus} s B_{1}$ and $A_{2} \lesssim_{\oplus} s B_{2}$, or $B_{1} \lesssim_{\oplus} s A_{1}$ and $B_{2} \lesssim_{\oplus} s A_{2}$, the assertion is obviously true. Now assume 
that $A_{1} \lesssim \oplus s B_{1}$ and $B_{2} \lesssim_{\oplus} s A_{2}$. By Lemma 1.5, there are a direct summand $V$ of $A_{1}$ such that $V \lesssim \oplus B_{1}$ and $A_{1} \lesssim \oplus s V$ and a direct summand $U$ of $B_{2}$ such that $U \lesssim_{\oplus} A_{2}$ and $B_{2} \lesssim_{\oplus} s U$. So $B_{1} \cong V \oplus C$ and $A_{2} \cong U \oplus D$ where $C$ and $D$ are finitely generated projective right $R$-modules. Since $C, D \lesssim_{\oplus}(n-1) R$, by the induction, we get $C \lesssim \oplus s D$, or $D \lesssim \oplus s C$. If the former is true, then

$$
\begin{aligned}
B=B_{1} \oplus B_{2} & \cong V \oplus C \oplus B_{2} \\
& \lesssim \oplus \oplus s D \oplus s U \\
& \cong V \oplus s A_{2} \\
& \lesssim \oplus A_{1} \oplus s A_{2} \\
& =s A .
\end{aligned}
$$

Similarly, if the latter is true, then $A \lesssim_{\oplus} s B$, as desired.

In order to use easily later, we give the following result which is well-known for regular rings [12, Lemma 1.2].

Proposition 1.7. Let $R$ be a refinement ring satisfying almost comparability.

(1) If $S$ is a simple projective $R$-module, then $S \lesssim_{\oplus} A$ for any nonzero finitely generated projective $R$-module $A$.

(2) All simple projective $R$-modules are isomorphic.

Proof. (1) Since $R$ satisfies almost comparability, it has 2-comparability, hence $S \lesssim_{\oplus} 2 A$, or $A \lesssim_{\oplus} 2 S$, by Proposition 1.6. If $S \lesssim_{\oplus} 2 A$, since $R$ has refinement, $S$ has decomposition $S=S_{1} \oplus S_{2}$ such that $S_{i} \lesssim_{\oplus} A, i=1,2$ by Lemma 1.4. Since $S$ is simple, $S_{1}=0$, or $S_{2}=0$. If $S_{1}=0$, then $S=S_{2} \lesssim \oplus A$. If $S_{2}=0$, then $S=S_{1} \lesssim_{\oplus} A$. If $A \lesssim_{\oplus} 2 S$, since $R$ has refinement, $A$ has decomposition

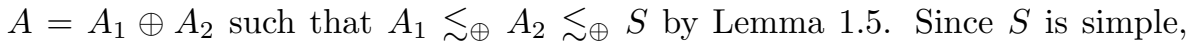
$A_{1}=0$, or $A_{2}=0$. If $A_{2}=0$, then $S=0$. If $A_{1}=0$, then $A=A_{2} \cong S$. So we have $S \lesssim \oplus$.

(2) Let $A, B$ be two simple projective $R$-modules, by (1), we have that $A \lesssim \oplus$. So $A \cong B$ as $B$ is simple.

Corollary 1.8. Let $R$ be an exchange ring satisfying almost comparability. The statements in Proposition 1.7 hold.

In 2007, Kutami [12, Proposition 1.3] proved that over any regular ring $R$, almost comparability and strictly almost comparability of every finitely generated projective $R$-module are equivalent. Actually, they coincide with each other over any ring.

Proposition 1.9. Let $R$ be a ring. For each finitely generated projective $R$ module $P$, the following are equivalent:

(1) $P$ has almost comparability;

(2) $P$ has strictly almost comparability.

Proof. $(2) \Rightarrow(1)$ is clear. 
$(1) \Rightarrow(2)$ For any direct summands $A$ and $B$ of $P$, by hypothesis, $A \lesssim_{a} B$ or $B \lesssim_{a} A$. If $A \lesssim_{a} B$, for any nonzero principal right ideal $C$ of $R, A \lesssim \oplus B \oplus C$. If there is a nonzero principal right ideal $D$ such that $A \cong B \oplus D$, then for any nonzero principal right ideal $E$ of $R$, we have $B \prec_{\oplus} A \prec_{\oplus} A \oplus E$. In this case, $B \prec_{a} A$. Otherwise, $A \prec_{a} B$.

Lemma 1.10. Let $R$ be a refinement ring satisfying almost comparability. And let $A, B$ be nonzero finitely generated projective $R$-modules. Then there exists a nonzero principal right ideal $X$ of $R$ such that $X \lesssim_{\oplus} A$, and $X \lesssim_{\oplus} B$.

Proof. At first, we argue that every finitely generated projective $R$-module has a nonzero cyclic submodule as a direct summand. Let $P$ be a finitely generated projective right $R$-module. There is another finitely generated projective right $R$-module $Q$ such that $P \oplus Q \cong R^{n}$ for some $n$. Since $R$ is a refinement ring, there are right $R$-modules $P_{1}, \ldots, P_{n}$ and $Q_{1}, \ldots, Q_{n}$ such that $P \cong P_{1} \oplus \cdots \oplus P_{n}$ and $Q \cong Q_{1} \oplus \cdots \oplus Q_{n}$ and $P_{i} \oplus Q_{i} \cong R$ for all $i$. Thus $P$ has a nonzero cyclic submodule isomorphic to, for example, $P_{1}$ as a direct summand. Now we can assume that both $A$ and $B$ are nonzero cyclic projective $R$-modules. Since $R$ satisfies almost comparability, $A \lesssim_{a} B$, or $B \lesssim_{a} A$. Without loss of generality, we assume that $A \lesssim a B$. Thus $A \lesssim \oplus 2 B$. Since $R$ is a refinement ring, $A$ has a decomposition $A=A_{1} \oplus A_{2}$ such that $A_{2} \lesssim_{\oplus} A_{1} \lesssim_{\oplus} B$ by Lemma 1.5. Let $X$ be a nonzero principal right ideal of $R$ such that $X \cong A_{1}$. Then we have $X \lesssim \oplus A$, and $X \lesssim \oplus B$.

Corollary 1.11. Let $R$ be an exchange ring satisfying almost comparability, and let $A$ and $B$ be nonzero finitely generated projective right $R$-modules. Then there exists a nonzero principal right ideal $X$ of $R$ such that $X \lesssim_{\oplus} A$, and $X \lesssim_{\oplus} B$.

Lemma 1.12 ([14, Theorem 1.9]). Let $R$ be a refinement ring satisfying $s$ comparability. Then $R$ is separative.

The following lemma for regular rings is first proved by Kutami [12, Lemma 1.7]. For the readers' convenience, we prove it here.

Lemma 1.13. Let $R$ be a refinement ring and assume that $n R$ satisfies almost comparability for some positive integer $n$. Let $A, B, C, D$ be finitely generated projective $R$-modules isomorphic to direct summands of $n R$. If $A \prec_{a} C$ and $B \prec_{a} D$, then $A \oplus B \prec_{a} C \oplus D$.

Proof. We may assume that $A \neq 0$. Let $X$ be any nonzero principal right ideal of $R$. Since $A \prec_{a} C$, we have $A \prec_{\oplus} C \oplus X$. Hence there is a nonzero finitely generated projective $R$-module $Y$ such that $A \oplus Y \cong C \oplus X$. By Lemma 1.10, since $R$ satisfies almost comparability, we get a nonzero principal right ideal $Y^{*}$ of $R$ such that $Y^{*} \lesssim_{\oplus} Y$, and $Y^{*} \lesssim_{\oplus} A$. Further, we have that $B \prec_{\oplus} D \oplus Y^{*}$ as $B \prec_{a} D$. Thus

$$
A \oplus B \oplus Y^{*} \lesssim \oplus A \oplus B \oplus Y \cong B \oplus C \oplus X \prec_{\oplus} C \oplus X \oplus D \oplus Y^{*} .
$$


Since $R$ is separative by Lemma 1.12, and $Y^{*} \lesssim_{\oplus} A$ and $Y^{*} \lesssim_{\oplus} Y \lesssim_{\oplus} C \oplus X$, we have that $A \oplus B \prec_{\oplus} C \oplus X \oplus D$ by [3, Lemma 2.1], i.e., $A \oplus B \prec_{a} C \oplus D$, as desired.

Corollary 1.14. Let $R$ be an exchange ring satisfying almost comparability, and let $A$ and $B$ be nonzero finitely generated projective right $R$-modules. If $A \prec_{a} C$ and $B \prec_{a} D$, then $A \oplus B \prec_{a} C \oplus D$.

Proof. By [3, Proposition 1.2], $R$ is a refinement ring. From Lemma 1.13, the assertion is true.

Theorem 1.15. Let $R$ be a refinement ring, and assume that $n R$ satisfies almost comparability for some positive integer $n$. Let $A, B, C, D$ be finitely generated projective $R$-modules isomorphic to direct summands of $n R$. If $A \prec_{a}$ $C$ and $D \prec{ }_{a} B$, then $A \oplus B \prec_{a} C \oplus D$ or $C \oplus D \prec{ }_{a} A \oplus B$.

Proof. (1) If $A=C=0$ or $B=D=0$, then the result obviously holds.

(2) If $A=D=0$, either $B \prec_{a} C$ or $C \prec_{a} B$ by the hypothesis of almost comparability of $n R_{R}$, as desired. Similarly, the result also holds in case $B=$ $C=0$.

(3) Either $A \neq 0$ or $B \neq 0$, and either $C \neq 0$ or $D \neq 0$. We consider the following four cases:

Case 1. $B \prec_{\oplus} n R_{R}$ and $C \prec_{\oplus} n R_{R}$;

Case 2. $B \cong n R_{R}$ and $C \cong n R_{R}$;

Case 3. $B \prec_{\oplus} n R_{R}$ and $C \cong n R_{R}$;

Case 4. $B \cong n R_{R}$ and $C \prec_{\oplus} n R_{R}$.

Case 1. There exist nonzero finitely generated projective $R$-modules $E$ and $F$ such that $B \oplus E \cong n R_{R}$ and $C \oplus F \cong n R_{R}$. Since $A \prec_{a} C$ and $D \prec_{a} B$, by Lemma 1.10, we can take a nonzero principal right ideal $X$ of $R$ such that $X$ is isomorphic to a direct summand of $A, C, E$, and $F$, and hence $A \prec_{\oplus} C \oplus X$ and $D \prec_{\oplus} B \oplus X$. So, there exist $Y$ and $Z$ such that $A \oplus Y \cong C \oplus X$ and $D \oplus Z \cong B \oplus X$. It follows from $X \lesssim_{\oplus} F$ and $X \lesssim_{\oplus} E$ that $C \oplus X \lesssim_{\oplus} n R_{R}$ and $B \oplus X \lesssim_{\oplus} n R_{R}$. Hence $Y$ and $Z$ are finitely generated projective $R$-modules which are isomorphic to direct summands of $n R_{R}$. By the hypothesis of almost comparability of $n R_{R}$, we have that either $Y \prec_{a} Z$ or $Z \prec_{a} Y$. Assume that $Y \prec_{a} Z$. Then, $C \oplus X \oplus D \cong A \oplus D \oplus Y \prec_{a} A \oplus D \oplus Z \cong A \oplus B \oplus X$. Note that $X \lesssim_{\oplus} A$ and $X \lesssim_{\oplus} C$, as $R$ is separative by Lemma 1.12, then $C \oplus D \prec_{a} A \oplus B$ by [3, Lemma 2.1$]$.

Case 2. We have that $B \cong C$. Since $n R_{R}$ satisfies almost comparability, either $A \prec_{a} D$ or $D \prec_{a} A$. Therefore, either $A \oplus B \prec_{a} C \oplus D$ or $C \oplus D \prec_{a} A \oplus B$.

Case 3. If $A \cong n R_{R}$, then $D \prec_{a} B$ implies $C \oplus D \prec_{a} A \oplus B$, as desired. Therefore, we can assume that $A \prec_{\oplus} n R_{R}$. There exist nonzero finitely generated projective $R$-modules $X$ and $C^{*}$ such that $C=X \oplus C^{*}$, where $X \cong A$. Since $D \prec_{a} B$ and $B \prec_{\oplus} n R_{R}$, by Lemma 1.10, there exists a nonzero principal right ideal $X^{*}$ of $R$ such that $X^{*} \lesssim_{\oplus} X, X^{*} \lesssim_{\oplus} A$, and $D \lesssim_{\oplus} B \oplus X^{*} \lesssim_{\oplus} n R_{R}$. Thus, we have a nonzero finitely generated projective $R$-module $Z$ such that 
$D \oplus Z \cong B \oplus X^{*}$. Set $Y=X^{*} \oplus C^{*}$, so $Y \lesssim \oplus n R_{R}$ and $Z \lesssim \oplus n R_{R}$. Since $n R_{R}$ satisfies almost comparability, either $Y \prec_{a} Z$ or $Z \prec_{a} Y$. Assume that $Y \prec_{a} Z$. Then $C \oplus D \oplus X^{*} \cong X \oplus D \oplus C^{*} \oplus X^{*} \cong A \oplus D \oplus Y \prec_{a} A \oplus D \oplus Z \cong A \oplus B \oplus X^{*}$. Since $R$ is separative, and $X^{*} \lesssim_{\oplus} C$ and $X^{*} \lesssim_{\oplus} A$, we see that $C \oplus D \prec_{a} A \oplus B$ by [3, Lemma 2.1].

Case 4. The same as the proof of the case 3 .

Corollary 1.16. Let $R$ be an exchange ring, and assume that $n R$ satisfies almost comparability for some positive integer $n$. Let $A, B, C, D$ be finitely generated projective $R$-modules isomorphic to direct summands of $n R$. If $A \prec_{a}$ $C$ and $D \prec_{a} B$, then $A \oplus B \prec_{a} C \oplus D$ or $C \oplus D \prec_{a} A \oplus B$.

Theorem 1.17. Let $R$ be a refinement ring. Then the following conditions are equivalent:

(1) $R$ satisfies almost comparability;

(2) Every finitely generated projective $R$-module satisfies almost comparability;

(3) $n R_{R}$ satisfies almost comparability for all positive integers $n$;

(4) $n R_{R}$ satisfies almost comparability for some positive integer $n$.

Proof. $(3) \Rightarrow(2) \Rightarrow(4) \Rightarrow(1)$ are clear.

$(1) \Rightarrow(3)$. Assume that $R$ satisfies almost comparability. We shall prove (3) by using the induction on $n$. We assume that $(n-1) R_{R}$ satisfies almost comparability, and let $V$ and $W$ be any direct summands of $n R_{R}$. Then there exist decompositions $V=A \oplus B$ and $W=C \oplus D$ such that all $A, B, C$, and $D$ are isomorphic to direct summands of $(n-1) R_{R}$. From Lemma 1.13 and Theorem 1.15, we see that either $V \prec_{a} W$ or $W \prec_{a} V$. Therefore, $n R_{R}$ satisfies almost comparability.

Proposition 1.18. Let $M$ be a finitely generated projective $R$-module over a refinement ring $R$, and set $S=\operatorname{End}_{R}(M)$. Then $M$ satisfies almost comparability if and only if so does $S$ as an $S$-module.

Proof. $\Rightarrow$ Let $A, B$ be summands of $S$. By [1, Lemma 29.4], $A \otimes_{S} M, B \otimes_{S} M$ are summands of $M_{R}$. Since $M$ satisfies almost comparability, $A \otimes_{S} M \lesssim_{a} B \otimes_{S} M$, or $B \otimes_{s} M \lesssim_{a} A \otimes_{S} M$. Assume that $A \otimes_{S} M \lesssim_{a} B \otimes_{S} M$. For any nonzero principal right ideal $C \lesssim_{\oplus} S$, we have $A \otimes_{S} M \lesssim_{\oplus}\left(B \otimes_{S} M\right) \oplus\left(C \otimes_{S} M\right)$. Thus there is a right $R$-module $X$ such that $\left(A \otimes_{S} M\right) \oplus X \cong\left(B \otimes_{S} M\right) \oplus\left(C \otimes_{S} M\right)$. Hence $\operatorname{Hom}_{R}\left(M,\left(A \otimes_{S} M\right) \oplus X\right) \cong \operatorname{Hom}_{R}\left(M,\left(B \otimes_{S} M\right) \oplus\left(C \otimes_{S} M\right)\right)$. That is, $\operatorname{Hom}_{R}\left(M, A \otimes_{S} M\right) \oplus \operatorname{Hom}_{R}(M, X) \cong \operatorname{Hom}_{R}\left(M, B \otimes_{S} M\right) \oplus \operatorname{Hom}_{R}\left(M, C \otimes_{S}\right.$ $M)$. Thus by using [1, Lemma 29.4] again, we have that $A \oplus \operatorname{Hom}_{R}(M, X) \cong$ $B \oplus C$, i.e., $A \prec_{a} B$.

$\Leftarrow$ The same as the above.

Theorem 1.19. Let $R$ be a refinement ring. The following conditions are equivalent:

(1) $R$ satisfies almost comparability; 
(2) For each finitely generated projective $R$-module $P, \operatorname{End}_{R}(P)$ satisfies almost comparability;

(3) Every ring $S$ which is Morita equivalent to $R$ satisfies almost comparability;

(4) For all positive integers $n, M_{n}(R)$ satisfies almost comparability;

(5) There exists a positive integer $n$ such that $M_{n}(R)$ satisfies almost comparability.

Proof. (1) $\Leftrightarrow(2)$ By Theorem 1.17, and Proposition 1.18, $R$ satisfies almost comparability if and only if every finitely generated projective $R$-module $P$ satisfies almost comparability, if and only if $\operatorname{End}_{R}(P)$ satisfies almost comparability.

$(1) \Leftrightarrow(3)$ Let $S$ be a ring which is Morita equivalent to $R$, and let $F$ and $G$ be inverse equivalences of the categories of $R$-modules and $S$-modules. By Theorem 1.17, $R$ satisfies almost comparability if and only if every finitely generated projective $R$-module $P$ satisfies almost comparability, and if and only if $F(P)$ satisfies almost comparability, and if and only if $S$ satisfies almost comparability.

$(1) \Leftrightarrow(4),(1) \Leftrightarrow(5)$ Since $R$ is Morita equivalent to $M_{n}(R)$, the assertions are true by $(1) \Leftrightarrow(3)$.

We remark that Theorems 1.15, 1.17 and 1.19 are motivated by [12, Propostion 1.8, Thereom 1.9 and Thereom 1.11], respectively. The assertions of Theorem 1.17, Proposition 1.18 and Theorem 1.19 are true if the ring $R$ is an exchange ring, since any exchange ring is a refinement ring by $[3$, Proposition $1.2]$.

\section{Cancellation property of refinement rings}

Recall that a module $M$ is called directly finite if $M$ is not isomorphic to any proper direct summand of itself, otherwise, $M$ is called directly infinite. A ring $R$ is said to be directly finite provided $R_{R}$ is a directly finite module. $R$ is called stably finite if all finitely generated projective $R$-modules are directly finite. It is well-known that for some positive integer $s$ refinement rings satisfying scomparability have cancellation of separativity [14, Theorem 1.9], and that directly finite refinement rings satisfying s-comparability have cancellation of strong separativity [14, Corollary 1.11]. In [12, Proposition 2.1], Kutami proved that for any regular ring satisfying almost comparability $A \oplus C \prec_{\oplus} B \oplus C$ implies that $A \prec_{\oplus} B$ for any nonzero finitely generated directly finite projective $R$-modules $A, B, C$. We consider the similar property of refinement rings. In order to do this, we need the following lemma which is well-known for regular rings.

Lemma 2.1. Let $R$ be a directly finite refinement ring satisfying s-comparability for some positive integer s. Then $R$ is stably finite. 
Proof. Since $R$ is a refinement ring satisfying s-comparability, by [14, Theorem 1.9], $R$ is separative. Since $R$ is directly finite, then by [3, Proposition 2.3] $R$ is stably finite.

Theorem 2.2. Let $R$ be a directly finite refinement ring satisfying almost comparability. Then $A \oplus C \oplus X \cong B \oplus C$ implies that $A \prec_{\oplus} B$ for any nonzero finitely generated projective $R$-modules $A, B, C$ and any simple projective $R$ module $X$.

Proof. At first, note that $R$ is stably finite by Lemma 2.1. Since $R$ satisfies almost comparability, by Proposition 1.7, $X \lesssim_{\oplus} A$. Let $X \oplus A^{*} \cong A$ for some finitely generated projective $R$-module $A^{*}$. So $A^{*} \oplus C \oplus 2 X \cong B \oplus C$. By Lemma 1.10, there is a nonzero principal right ideal $X^{*}$ of $R$ such that $X^{*} \lesssim \oplus$ $X, A^{*}$ and $B$. Because $R$ satisfies almost comparability, by Theorem 1.17 and Proposition 1.9, either $B \prec_{a} A^{*} \oplus X^{*}$ or $A^{*} \oplus X^{*} \prec_{a} B$. If $B \prec_{a} A^{*} \oplus X^{*}$, then $A^{*} \oplus C \oplus X \oplus X^{*} \lesssim \oplus A^{*} \oplus C \oplus 2 X \cong B \oplus C \prec A^{*} \oplus C \oplus X^{*} \oplus X$, which contradicts the direct finiteness of $A^{*} \oplus C \oplus X^{*} \oplus X$, since $R$ is stably finite. So we have $A^{*} \oplus X^{*} \prec_{a} B$. And then $A^{*} \oplus X^{*} \prec_{\oplus} B \oplus X^{*}$. Since $R$ is separative by [14, Theorem 1.9] and $X^{*} \lesssim_{\oplus} A^{*}, B$, we have $A^{*} \prec_{\oplus} B$ by [3, Lemma 2.1]. Let $A^{*} \oplus Y \cong B$ for some nonzero finitely generated projective $R$-module $Y$. By Proposition 1.7, we have $X \lesssim \oplus Y$. Thus $A \cong X \oplus A^{*} \lesssim \oplus A^{*} \oplus Y \cong B$. If $A \cong B$, it follows from $A \oplus \widetilde{C} \oplus X \cong B \oplus C$ that $B \oplus \widetilde{C} \oplus X \cong B \oplus C$, which contradicts the direct finiteness of $B \oplus C$, since $R$ is stably finite. Thus $A \prec_{\oplus} B$.

Recall that a ring has cancellation of the strict unperforation property provided that $n A \prec_{\oplus} n B$ implies $A \prec_{\oplus} B$ for any finitely generated projective $R$-modules $A$ and $B$. Ara et al. [5, Example 4.11] showed that unit-regular rings with 2-comparability do not necessarily have cancellation of the strictly unperforation property. But some directly finite refinement rings satisfying almost comparability have the cancellation property (Theorem 2.4).

Lemma 2.3. Let $R$ be a refinement ring satisfying almost comparability with a simple right ideal $S \lesssim_{\oplus} R$. If $A \prec_{a} B$, then $A \lesssim_{\oplus} B$ for any nonzero finitely generated projective $R$-modules $A$ and $B$.

Proof. By Proposition 1.7, $S \lesssim \oplus A$ and $S \lesssim \oplus B$. Let $A \cong S \oplus A^{*}, B \cong S \oplus B^{*}$ for some finitely generated projective $R$-modules $A^{*}$ and $B^{*}$. If $A^{*}=0$, it is clear that $A \lesssim_{\oplus} B$. So we may assume that $A^{*} \neq 0$. Note that $A \prec_{a} B$, then we have $S \oplus A^{*} \cong A \prec \oplus S \oplus B$. Since $R$ is separative by [14, Theorem 1.9] and $S \lesssim_{\oplus} A$ and $S \lesssim_{\oplus} B$, by [3, Lemma 2.1], we have that $A^{*} \prec_{\oplus} B$. Let $B \cong A^{*} \oplus X$ for some nonzero finitely generated projective $R$-module $X$. So $A \cong S \oplus A^{*}{\lesssim_{\oplus}}^{*} \oplus X \cong B$, i.e., $A \lesssim_{\oplus} B$.

We remark that there are plenty of examples of rings which have a simple right ideal as a direct summand but which are not regular. For example, if $V$ is an infinite dimensional vector space over a field, $E$ is the ring of all linear 
transformations on $V$, and $J$ is the set of linear transformations in $E$ with finite rank (i.e., having finite dimensional image), then any subring $R$ of $E$ which contains $J$ satisfies the conditions. The author thanks K. R. Goodearl for his giving the example.

Theorem 2.4. Let $R$ be a directly finite refinement ring satisfying almost comparability with a simple right ideal $S \lesssim_{\oplus} R$. For any two nonzero finitely generated projective $R$-modules $A$ and $B$ and positive integer $n, n A \prec_{\oplus} n B$ implies $A \prec_{a} B$.

Proof. Since $R$ satisfies almost comparability, either $A \prec_{a} B$ or $B \prec_{a} A$, by Theorem 1.17 and Proposition 1.9. Assume that the latter is true. By Lemma $2.3, B \lesssim_{\oplus} A$. Let $B \oplus C \cong A$ for some finitely generated projective $R$-module $C$. So $n B \oplus n C \cong n A$. Since $n A \prec \oplus n B$, we have $n A \oplus D \cong n B$ for some nonzero finitely generated projective $R$-module $D$. Therefore, $n A \oplus D \oplus n C \cong n A$, which contradicts the direct finiteness of $n A$, since $R$ is stably finite.

Recall that a ring $R$ is said to satisfy weak comparability if for each nonzero $x \in R$, there is $n=n(x)$ such that $n(y R) \lesssim_{\oplus} R$ implies that $y R \lesssim_{\oplus} x$ for all $y \in R$. There exists a non-simple directly infinite refinement ring with both 1-comparability (hence almost comparability) and weak comparability $[11$, p. 3145]. For the cancellation property of special case of exchange rings with weak comparability, we have the following results.

Theorem 2.5. Let $R$ be an exchange ring with weak comparability. Then $A \oplus C \lesssim \oplus B \oplus C$ implies $A \lesssim \oplus B$ for any nonzero finitely generated projective $R$-modules $A, B$ and $C$ with $B$ directly infinite.

Proof. By [15, Theorem 1], we may assume that $C$ is cyclic. Since $B$ is directly infinite, there is a nonzero cyclic projective $R$-module $W$ such that $B \oplus W \lesssim \oplus$ $B$. Since $R$ satisfies weak comparability, there is a positive integer $m$ such that $m X \lesssim \oplus R$ implies that $X \lesssim \oplus W$ for any cyclic projective $R$-module $X$. Since $A \oplus C \lesssim \oplus B \oplus C$, we have a decomposition $B \oplus C=A^{\prime} \oplus C^{\prime} \oplus D$ for some projective $R$-modules $A^{\prime}, C^{\prime}$ and $D$ such that $A^{\prime} \cong A, C^{\prime} \cong C$. Let $f$ be the isomorphism from $C$ to $C^{\prime}$. Since $R$ is an exchange ring, the finitely generated projective $R$-module $A^{\prime}$ has the exchange property. Thus there exist decompositions $B=B_{1} \oplus B_{1}^{*}$ and $C=C_{1} \oplus C_{1}^{*}$ such that $B \oplus C=A^{\prime} \oplus B_{1}^{*} \oplus C_{1}^{*}$. And so $A^{\prime} \cong B_{1} \oplus C_{1}$. Since $A^{\prime} \oplus f C_{1}$ is the direct summand of $B \oplus C$, it has the exchange property. Thus there are decompositions $B_{1}^{*}=B_{2} \oplus B_{2}^{*}$ and $C_{1}^{*}=C_{2} \oplus C_{2}^{*}$ such that $B \oplus C=A^{\prime} \oplus f C_{1} \oplus B_{2}^{*} \oplus C_{2}^{*}$. So $f C_{1} \cong B_{2} \oplus C_{2}$. Since $C_{1} \cap C_{2}=0$, we have that $A^{\prime} \oplus f C_{1} \oplus f C_{2}$ is a direct summand of $B \oplus C$. Continuing the above procedure, we have decompositions $B_{n}^{*}=B_{n+1} \oplus B_{n+1}^{*}$ and $C_{n}^{*}=C_{n+1} \oplus C_{n+1}^{*}$ such that $B \oplus C=A^{\prime} \oplus f C_{1} \oplus \cdots \oplus f C_{n} \oplus B_{n+1}^{*} \oplus C_{n+1}^{*}$ and $f C_{n} \cong B_{n+1} \oplus C_{n+1}$. If $C_{k}=0$ for some positive integer $k$, we have that $A \cong A^{\prime} \cong B_{1} \oplus C_{1} \cong B_{1} \oplus f C_{1} \cong B_{1} \oplus B_{2} \oplus C_{2} \cong \ldots \cong B_{1} \oplus B_{2} \oplus \cdots \oplus B_{k-1} \oplus$ $C_{k-1} \cong B_{1} \oplus B_{2} \oplus \cdots \oplus B_{k} \leq_{\oplus} B$. If $C_{n} \neq 0$ for all positive integers $n$, since 
$C_{m} \lesssim_{\oplus} \cdots \lesssim_{\oplus} C_{1} \leq_{\oplus} C$, we have that $m C_{m} \lesssim_{\oplus} C \lesssim_{\oplus} R$, and so $C_{m} \lesssim_{\oplus} W$. Thus we have that

$$
\begin{aligned}
A \cong A^{\prime} \cong B_{1} \oplus C_{1} & \cong B_{1} \oplus f C_{1} \cong B_{1} \oplus B_{2} \oplus C_{2} \\
& \cong \cdots \cong B_{1} \oplus B_{2} \oplus \cdots \oplus B_{m} \oplus C_{m} \\
& \lesssim \oplus B_{1} \oplus B_{2} \oplus \cdots \oplus B_{m} \oplus W \\
& \leqslant \oplus B \oplus W \lesssim_{\oplus} B
\end{aligned}
$$

and we complete the proof.

Theorem 2.6. Let $R$ be an exchange ring with weak comparability. Then $A \oplus C \prec_{\oplus} B \oplus C$ implies $A \prec_{\oplus} B$ for any nonzero finitely generated projective $R$-modules $A, B$ and $C$, if $C$ has the property that $\aleph_{0} X Z_{\oplus} C$ for any nonzero finitely generated projective $R$-module $X$.

Proof. By [15, Theorem 1], we may assume that $C$ is cyclic. Since $A \oplus C \prec_{\oplus}$ $B \oplus C$, we have a decomposition $B \oplus C=A^{\prime} \oplus C^{\prime} \oplus D$ for some nonzero projective $R$-modules $A^{\prime}, C^{\prime}$ and $D$ such that $A^{\prime} \cong A, C^{\prime} \cong C$. Let $f$ be the isomorphism from $C$ to $C^{\prime}$. Since $R$ is an exchange ring, the finitely generated projective $R$-module $D$ has the exchange property. Thus there exist decompositions $B=$ $B_{1} \oplus B_{1}^{*}$ and $C=C_{1} \oplus C_{1}^{*}$ such that $B \oplus C=D \oplus B_{1}^{*} \oplus C_{1}^{*}$. And so $D \cong B_{1} \oplus C_{1}$. Since $D \oplus f C_{1}$ is isomorphic to the direct summand of $B \oplus C$, it has the exchange property. Thus there are decompositions $B_{1}^{*}=B_{2} \oplus B_{2}^{*}$ and $C_{1}^{*}=C_{2} \oplus C_{2}^{*}$ such that $B \oplus C=D \oplus f C_{1} \oplus B_{2}^{*} \oplus C_{2}^{*}$. So $C_{1} \cong f C_{1} \cong B_{2} \oplus C_{2}$. Since $C_{1} \cap C_{2}=0$, we have that $D \oplus f C_{1} \oplus f C_{2}$ is isomorphic to a direct summand of $B \oplus C$. Continuing the above procedure, we have decompositions $B_{n}^{*}=B_{n+1} \oplus B_{n+1}^{*}$ and $C_{n}^{*}=C_{n+1} \oplus C_{n+1}^{*}$ such that $B \oplus C=D \oplus f C_{1} \oplus \cdots \oplus f C_{n} \oplus B_{n+1}^{*} \oplus C_{n+1}^{*}$ and $C_{n} \cong f C_{n} \cong B_{n+1} \oplus C_{n+1}$ for each $n=1,2, \ldots$. We claim that $B_{k} \neq 0$ for some positive integer $k$. In fact, if $B_{k}=0$ for all $k, 0 \neq \aleph_{0} D \cong C_{1} \oplus C_{2} \oplus \cdots \leq_{\oplus}$ $C$, which contradicts the hypothesis. Since $B \oplus C=A^{\prime} \oplus C^{\prime} \oplus D$, we have that $A^{\prime} \oplus f C_{k}^{*} \oplus\left(D \oplus f C_{1} \oplus \cdots \oplus f C_{k-1}\right) \leq_{\oplus} A^{\prime} \oplus f C_{1} \oplus \cdots \oplus f C_{k-1} \oplus$ $f C_{k} \oplus f C_{k}^{*} \oplus D=B_{k}^{*} \oplus C_{k}^{*} \oplus\left(D \oplus f C_{1} \oplus \cdots \oplus f C_{k-1}\right)$. Since $A^{\prime} \oplus f C_{k}^{*}$ is a finitely generated projective $R$-module, according to the above procedure, there exist decompositions $B_{k}^{*}=B_{k 1} \oplus B_{k 1}^{*}, \ldots, B_{k n}^{*}=B_{k, n+1} \oplus B_{k, n+1}^{*}$ and $C_{k}^{*}=C_{k 1} \oplus C_{k 1}^{*}, \ldots, C_{k n}^{*}=C_{k, n+1} \oplus C_{k, n+1}^{*}$ such that $A^{\prime} \cong B_{k 1} \oplus C_{k 1}$ and $C_{k n} \cong B_{k, n+1} \oplus C_{k, n+1}$. Then we have that $n C_{k n} \lesssim \oplus C \lesssim \oplus$ for each positive integer $n$. If $C_{k m}=0$ for some $m$, then

$$
\begin{aligned}
A \cong A^{\prime} \cong B_{k 1} \oplus C_{k 1} & \cong B_{k 1} \oplus B_{k 2} \oplus C_{k 2} \\
& \cong \cdots \cong B_{k 1} \oplus \cdots \oplus B_{k, m-1} \oplus C_{k, m-1} \\
& \cong B_{k 1} \oplus \cdots \oplus B_{k m} \\
& \prec \oplus B_{k} \oplus B_{k 1} \oplus \cdots \oplus B_{k m} \\
& \leqslant \oplus B .
\end{aligned}
$$

If $C_{k m} \neq 0$ for all positive integers $m$. Since $B_{k} \neq 0$, there is a nonzero cyclic submodule $Z$ of $B_{k}$ as a direct summand. Thus using the weak comparability 
for $R$, we have that $C_{k l} \lesssim_{\oplus} Z \leq_{\oplus} B_{k}$ for some positive integer $l$. We claim that $B_{k s} \neq 0$ for some positive integer $s>l$. In fact, if $B_{k s}=0$ for all positive integers $s>l$, then $C_{k l} \cong C_{k, l+1} \cong \cdots$ and $C_{k l} \oplus C_{k, l+1} \oplus \cdots \leqslant \oplus C$, so $\aleph_{0} C_{k l} \leq_{\oplus} C$, which contradicts the hypothesis. Thus

$$
\begin{aligned}
A \cong A^{\prime} \cong B_{k 1} \oplus C_{k 1} & \cong B_{k 1} \oplus B_{k 2} \oplus C_{k 2} \\
& \cong \cdots \cong B_{k 1} \oplus \cdots \oplus B_{k, l} \oplus C_{k, l} \\
& \prec \oplus B_{k} \oplus B_{k 1} \oplus \cdots \oplus B_{k l} \oplus B_{k s} \\
& \leq{ }_{\oplus} B .
\end{aligned}
$$

Remark 2.7. (1) In Kutami's 2005 paper, he proved that for a directly infinite regular ring $R$ with weak comparability, $A \oplus C \lesssim_{\oplus} B \oplus C$ implies $A \prec_{\oplus} B$ for any nonzero finitely generated projective $R$-modules $A, B$ and $C$ with $B \neq 0$, see [11, Proposition 1.5]. So the idea of Theorem 2.5 is due to Kutami.

(2) Recall that a module $M$ is said to have finite uniform dimension if it contains no infinite direct sum of nonzero submodules. It is clear that any module with finite uniform dimension (hence any uniform module and Noetherian module) has the hypothesis of Theorem 2.6.

(3) In 2003, Kutami proved that for a regular $\operatorname{ring} R$ with weak comparability, $A \oplus C \prec_{\oplus} B \oplus C$ implies $A \prec_{\oplus} B$ for any finitely generated projective $R$-modules $A, B$ and $C$ with $C$ directly finite, see [10, Theorem 2.2]. Theorem 2.6 is motivated by [10, Theorem 2.2].

We end this note by raising the following questions:

Question 2.8. (1) Does there exist a refinement ring with almost comparability which is not regular and without strictly unperforated property?

(2) Does there exist a refinement ring with weak comparability that is not a regular ring without almost comparability?

Acknowledgments. The author wishes to express their gratitude to the referee for his/her careful reading and comments which improve the presentation of this article and to Professor Xiaosheng Zhu for his helpful suggestions.

\section{References}

[1] F. W. Anderson and K. R. Fuller, Rings and Categories of Modules, New YorkHeidelberg-Berlin, Springer-Verlag, 1992.

[2] P. Ara and K. R. Goodearl, The almost isomorphism relation for simple regular rings, Publ. Mat. 36 (1992), 369-388.

[3] P. Ara, K. R. Goodearl, K. C. O'Meara, and E. Pardo, Separative cancellation for projective modules over exchange rings, Israel J. Math. 105 (1998), 105-137.

[4] _ Diagonalization of matrices over regular rings, Linear Algebra Appl. 265 (1997), 147-163.

[5] P. Ara, K. C. O'Meara, and D. V. Tyukavkin, Cancellation of projective modules over regular rings with comparability, J. Pure Appl. Algebra 107 (1996), no. 1, 19-38.

[6] P. Ara and E. Pardo, Refinement monoids with weak comparabiliy and applications to regular rings and $C^{*}$-algebras, Proc. Amer. Math. Soc. 124 (1996), no. 3, 715-720. 
[7] P. Crawley and B. Jónsson, Refinements for infinite direct decompositions of algebraic systems, Pacific J. Math. 14 (1964), 797-855.

[8] K. R. Goodearl, Von Neumann Regular Rings, Pitman, London, 1979.

[9] I. Kaplansky, Regular Banach algebras, J. Indian Math. Soc. (N.S.) 12 (1948), 57-62.

[10] M. Kutami, On von Neumann regular rings with weak comparability, J. Algebra 265 (2003), no. 1, 285-298.

[11] - On von Neumann regular rings with weak comparability. II, Comm. Algebra 33 (2005), no. 9, 3137-3147.

[12] - On regular rings satisfying almost comparability, Comm. Algebra 35 (2007), no. $7,2171-2182$

[13] H. X. Lin, An Introduction to the Classification of Amenable $C^{*}$-algebras, World Scientific Publishing Co. Pte. Ltd., Singapore, 2001.

[14] E. Pardo, Comparability, separativity, and exchange rings, Comm. Algebra 24 (1996), no. 9, 2915-2929.

[15] R. B. Warfield, Exchange rings and decompositions of modules, Math. Ann. 199 (1972), $31-36$

Department of Mathematics

NANJiNG University

NANJING 210093, P. R. ChINA

E-mail address: huangchaoling43@yahoo.com.cn 